\title{
On $q$-Deformed Supersymmetric Classical Mechanical Models
}

\author{
L.P. Colatto ${ }^{a, b *}$ and J.L. Matheus - Valle $^{b, c \dagger}$ \\ ${ }^{a}$ International Centre for Theoretical Physics, \\ Strada Costiera, 11, P. O. Box 586, 34100, Trieste, Italy; \\ ${ }^{b} \mathrm{CBPF} / \mathrm{CNPq}$, Rua Dr. Xavier Sigaud, 150 \\ 22290-180 Rio de Janeiro, RJ, Brazil \\ and \\ ${ }^{c}$ Departamento de Física, ICE \\ Universidade Federal de Juiz de Fora \\ 36036-330 Juiz de Fora, MG, Brazil
}

\begin{abstract}
Based on the idea of quantum groups and paragrassmann variables, we present a generalization of supersymmetric classical mechanics with a deformation parameter $q=\exp \frac{2 \pi i}{k}$ dealing with the $k=3$ case. The coordinates of the $q$-superspace are a commuting parameter $t$ and a paragrassmann variable $\theta$, where $\theta^{3}=0$. The generator and covariant derivative are obtained, as well as the action for some possible superfields.
\end{abstract}

MIRAMARE - TRIESTE

October 1995

*email: colatto@ictp.trieste.it

${ }^{\dagger}$ e-mail: zeluiz@cbpfsu1.cat.cbpf.br 


\section{Introduction}

In the last few years, Quasi Triangular Hopf Algebras or Quantum Groups [1, 2, 3] have attracted a lot of attention from physicists. One of the most interesting features is that such structures can be related to underlying symmetries on spaces where the coordinates are noncommutative 沟.

It has been shown that the creation and annihilation operators of the $q$-deformed harmonic oscillator [5]

$$
a a^{\dagger}-q a^{\dagger} a=q^{-N}
$$

possess a classical limit where these operators can be understood as coordinates obeying [6]

$$
\theta^{k}=0
$$

where $k$ is an integer, and the $q$-factor of the deformation is a prime root of unity, $q^{k}=1$. In general, the properties of these coordinates are generalizations of the associated with Grassmann variables. Promoting these coordinates to functions of a (non-deformed) parameter $t$, it was shown that it is possible to write down an action for such fields that, when added to the action of a commuting field, has a symmetry resembling supersymmetry [7], and it has also been how to functional integrate on a heterotic quantum field theory [8]. The aim of this letter is to show how a way to understand the transformations on such fields, and the action invariances, as resulting from a superspace formulation of a classical mechanical model where its coordinates are the paragrassmann variables (a $q$-superspace), and non-commuting fields.

In the next section we briefly review paragrassmann variables and also how we construct coordinates and actions from them. Section 3 is devoted to the construction of the $q$-superspace, transformations between its coordinates, and the induced transformations on the $q$ superfields defined on it. Invariant quadratic actions are constructed in Section 4, in particular for a free particle and the harmonic oscillator. We leave some final comments to the last section. 


\section{Paragrassmann Variables and their Relation to Quermionic Coordinates}

We start this section by introducing a paragrassmann variable $\theta$ and its derivative, $\frac{\partial}{\partial \theta} \equiv \partial_{\theta}$ obeying [9]

$$
\theta^{k}=0, \quad \partial_{\theta}^{k}=0
$$

for a positive integer $k$.

If we demand that the action of $\partial_{\theta}$ on $\theta^{n}$ is proportional to $\theta^{n-1}$, it turns out that it becomes necessary to deform the Leibnitz rule to be

$$
\partial_{\theta}(a b)=\left(\partial_{\theta} a\right) b+g(a)\left(\partial_{\theta} b\right)
$$

where $a, b$ are arbitrary polynomials in $\theta$, and $g(a)$ is an automorphism of the algebra, satisfying

$$
\begin{aligned}
g(\alpha a+\beta b) & =\alpha g(a)+\beta g(b), \\
g(a b) & =g(a) g(b),
\end{aligned}
$$

where $\alpha, \beta$ are c-numbers.

Choosing $a=\theta$ in (雨), we see that $\partial_{\theta}$ and $\theta$ must obey a $q$-deformed commutation (quommutation) relation

$$
\left[\partial_{\theta}, \theta\right]_{q} \equiv \partial_{\theta} \theta-q \theta \partial_{\theta}=1
$$

implying for $\theta$ the automorphism

$$
g(\theta)=q \theta .
$$

This derivative, however, is not unique. Indeed, we could change the power 1 in eq. (6) by any other integer, thus for each value of $k$ one can define $k-1$ different derivatives. For the specific case $k=3$, one may also define another derivative $\delta_{\theta}$ [10] that quommutes with $\theta$ as

$$
\left[\delta_{\theta}, \theta\right]_{q^{2}} \equiv \delta_{\theta} \theta-q^{2} \theta \delta_{\theta}=1,
$$

and its Leibnitz rule differs from eq. (4) by changing $g(a)$ to $g(g(a))$. These two derivatives have the following $q$-commutation relation

$$
\partial_{\theta} \delta_{\theta}-q \delta_{\theta} \partial_{\theta}=1
$$


As in the Grassmannian case, it is not possible to define the integral over $\theta$ as the inverse of the derivative. However, if we impose translation invariance and homogenity for the integral, it must be of the form

$$
\int d \theta \theta^{n} \alpha \delta_{n, k-1}
$$

It is interesting to notice that, for $k=2, q=-1$, eq.(11) becomes the usual anticommutator, consistent with eqs.(3) and (6), which are the conditions for Grassmann variables. Taking $k \rightarrow \infty$, eq.(11) becomes the usual commutator. The meaning of this limit in eq.(3) is that, if we Taylor expand a function of these variables, it will become a series (obviously, if $\theta^{k}=0, k$ finite, a Taylor expansion will be a polynomial of degree $(k-1))$, being

$$
f=a_{0}+\theta a_{1}+\theta^{2} a_{2}+\ldots+\theta^{k-1} a_{k-1}
$$

where we can promote the functions of a (commuting) parameter $t$.

Let us recall that in the Grassmannian case we have two different coordinates: one that behaves like $\theta$ (a fermionic coordinate), and another that behaves like $\theta^{0}$, a bosonic (commuting) coordinate. In the paragrassmann case, we will have $k$ different types of coordinates, each one corresponding to a power of $\theta$, and again $\theta^{0}$ being a commuting one. We call $\psi^{(i)}(t)$ the $q$-fermionic generalization of the coordinates or, simply, the quermionic coordinates and its label $(i)$ indicates the sector to which it belongs.

In a some recent work [7], it was emphasized that two quermions of different sectors obey the quommutation relation

$$
\psi^{(i)} \psi^{(j)}=q_{(i, j)} \psi^{(j)} \psi^{(i)},
$$

where the parameters $q_{(i, j)}$ are simply powers of $q, q^{k}=1$.

The particular case $k=3$ was taken, and an action which extends the supersymmetric point particle through the use of these generalized fields was constructed. This generalized particle was described by the coordinates $\left(x(t), \psi^{(1)}(t), \psi^{(2)}(t)\right)$, in the same way as a supersymmetric point particle is described by the coordinates $(x(t), \psi(t))$. The showed action involving the quermions was given by

$$
S=\int d t\left(\frac{1}{2} \dot{x}^{2}-q C^{(s)^{2}} \dot{\psi}^{(2)} \psi^{(1)}\right),
$$


with the mass equal to one. The second term in (12) resembles the classical fermionic equation of motion. The cocycle-type factor $C^{(s)^{2}}$ was required because the product of two objects of different sectors, $A^{(r)} B^{(s)}$, must behave like an object of the sector $(r+s) \bmod 3$. In that work, it was emphasized the necessity of the factor Cq-superfield, paying the price of writing a suitable "algebra" of this factors. For instance, the cocycle-type factor $C^{(s)}$ that could be seen as a sectorcounter, had a relation

$$
C^{(s)} A^{(i)}=q^{i} A^{(i)} C^{(s)},
$$

and adding the choice $\left[\psi^{(1)}, \psi^{(2)}\right]_{q}=0$, that take all the fields as real, the second term in the action eq. (12) was left real and a zeroth sector representative.

Another interesting feature to recall was the transformation (the variations of a field, from now on, will be written as $\Delta$ to one not to be confused with the derivative $\delta$ ),

$$
\begin{aligned}
\Delta x & =q C^{(s)} \epsilon^{(1)} \psi^{(2)}, \\
\Delta \psi^{(1)} & =q^{2} C^{(s)^{2}} \epsilon^{(1)} \dot{x}, \\
\Delta \psi^{(2)} & = \pm q \epsilon^{(1)} \psi^{(1)},
\end{aligned}
$$

on the action (12) reaching

$$
\Delta S= \pm \int d t \frac{d}{d t}\left(\epsilon^{(1)} \psi^{(1)^{2}}\right)
$$

where $\left[\epsilon^{(1)}, \psi^{(1)}\right]_{q}=\left[\psi^{(2)}, \epsilon^{(1)}\right]_{q}=0$ was used. Such transformation is similar to a supersymmetric one: the parameter $\epsilon^{(1)}$ is a noncommuting one, the action transforms as a total derivative, and one of the fields, $\psi^{(1)}$, transforms as a total derivative, which can be taken as indicating that $\psi^{(1)}$ is the highest term in a $\theta$-expansion of some superfield. One could also write transformations among the fields with a parameter belonging to the sector-two. However, it can be shown that this transformation is not a symmetry of the action (12) [7].

\section{The $q$-Superspace and $q$-Superfields}

We begin to construct a $q$-superspace formulation that will recover the structure concerning the quermionic coordinates presented in the 
last section. As previously stated, we will consider in detail only the $k=3$ case, that represents the nilpotency and produces an interesting expression $1+q+q^{2}=0$. It emphasizes that the $q$ and $q^{2}$ cases have not crucial difference. Some of the ideas discussed here and in the next section had been discussed also in refs. [11, 12] and more recently in [13].

The $q$-superspace coordinates are $(t ; \theta)$, where $t$ is a c-number to be identified with time and $\theta$ is a paragrassmannian variable obeying $\theta^{3}=0$, and both are taken as real parameters.

Let us now introduce transformations between these coordinates that are translations on the $q$-superspace. We write them as

$$
\begin{aligned}
\theta^{\prime} & =\theta+\varepsilon, \\
t^{\prime} & =t+q^{C} \theta^{2} \varepsilon,
\end{aligned}
$$

where $\varepsilon$ is an infinitesimal constant in the same sector as $\theta$ and $C$ can assume the values $1,2,3$. Clearly, the exponent 3 will give us a trivial factor restricting then our set of possible choices. The translation in $q$ superspace fixes the mass dimensions of $\theta$ and $\varepsilon$ to be $-\frac{1}{3}$. Although the translation term in $t$ does not commute with the infinitesimal parameter $\varepsilon$, it still belongs to the same sector as $t$. (Remember that we met this issue when we wrote down the action for the quermionic components, eq.(12) and we introduced the cocycle-like factor $C^{(s)}$ to correct the statistics). We will say that two terms are homogeneous if botthe same sector. Defining the quommutator to be

$$
[A, B]_{q} \equiv A B-q B A
$$

we choose

$$
[\theta, \varepsilon]_{q^{2 C}}=0
$$

It is after determining these quommutation relations that we set the $q$ factors in (16) to preserve the reality condition for the coordinates. We could choose $q^{C}$ instead of $q^{2 C}$ in (18) (i.e., take $[\varepsilon, \theta]_{q^{2 C}}=0$ ). With this choice we necessarily have to change $q \leftrightarrow q^{2}$ in eq. (16).

After introducing the $q$-superspace $(t, \theta)$, our next step is to write down a function of these variables. As in the supersymmetric case, let us expand this function in a Taylor series on $\theta$, this expansion is

\footnotetext{
${ }^{1}$ This nice work appeared when we was submitting this paper.
} 
a polynomial of degree 2 (for the generic case $\theta^{k}=0$, the polynomial goes up to the order $(k-1))$,

$$
X(t ; \theta)=x(t)+q^{B_{2}} \theta \psi^{(2)}(t)+q^{2 B_{1}} \theta^{2} \psi^{(1)}(t) .
$$

The coordinate $x(t)$ is a commuting function, the $\psi^{(i)}(t)$ are the $q$ supersymmetric partners of the coordinate $x(t)$, and their dimensions are $\left[\psi^{(j)}\right]=-\frac{j}{3}$. We take their quommutators to be

$$
\begin{aligned}
{\left[\psi^{(1)}, \psi^{(2)}\right]_{q^{A}} } & =0, \\
{\left[\varepsilon, \psi^{(j)}\right]_{q^{D_{j}}} } & =0, \\
{\left[\theta, \psi^{(j)}\right]_{q^{B_{j}}} } & =0,
\end{aligned}
$$

where the last expression guarantee that $X$ is real and the others complete a deformed algebra.

The infinitesimal coordinate transformations (16) induce a variation on the $q$-superfield $X(t, \theta)$ of the form

$$
X\left(t^{\prime}, \theta^{\prime}\right)-X(t, \theta)=\Delta X=\varepsilon Q X .
$$

We can get the realization of the q-supersymmetric generator transformation, $Q$, by Taylor expanding the l.r.s. of this equation. Choosing the factors to keep the reality condition we have

$$
X\left(\theta^{\prime}, t^{\prime}\right)-X(\theta, t)=\varepsilon \frac{\partial X}{\partial \theta}+q^{2 C} \varepsilon \theta^{2} \frac{\partial X}{\partial t},
$$

With this expansion, and using eq.(16), $Q$ becomes

$$
Q=q^{2 C} \theta^{2} \frac{\partial}{\partial t}+\frac{\partial}{\partial \theta} \text {. }
$$

We notice that the generator is in the $\theta^{2}$ sector, and its canonical dimension is $[Q]=\frac{1}{3}$. A straightforward calculation shows that $Q^{3}=$ $-\partial_{t}$. This means that the $q$-supersymmetric transformations are the cubic roots of time translations.

Explicitly computing the r.h.s. of (21), we obtain the $X$ variation as

$$
\Delta X=q^{B_{2}} \varepsilon \psi^{(2)}-q^{2 B_{1}+2+C} \theta \varepsilon \psi^{(1)}+q^{2 C} \theta^{2} \varepsilon \dot{x} .
$$

Bearing in mind the reality condition we find from $\Delta X$ and $X$ itself some relations among the $q$ exponents. Finally we reach

$$
\begin{array}{r}
2 C=2 B_{2}=B_{1}, \\
D_{2}=D_{1}+1 .
\end{array}
$$


The above relations do not fix completely the quommutators among the variables (see (201) we are considering, since we still have at our disposal three free coefficients. We may choose the variables $2 C=$ $D_{2}=1$ and $A=2$, thus fixing all the other ones (remember that the $k=3$ nilpotency we have only two relevant choices for the exponents). With such a choice, the $q$-superspace translation becomes

$$
\begin{array}{r}
\theta^{\prime}=\theta+\varepsilon, \\
t^{\prime}=t+q^{2} \theta^{2} \varepsilon,
\end{array}
$$

while the $q$-su

$$
X(t)=x+q^{2} \theta \psi^{(2)}+q^{2} \theta^{2} \psi^{(1)} .
$$

The $q$-SUSY generator

$$
Q=q \theta^{2} \partial_{t}+\partial_{\theta}
$$

yields the transformation

$$
\Delta X=q \varepsilon \theta^{2} \dot{x}+q^{2} \varepsilon \psi^{(2)}-q \varepsilon \theta \psi^{(1)},
$$

or in components

$$
\begin{array}{r}
\Delta x=q^{2} \varepsilon \psi^{(2)}, \\
\Delta \psi^{(1)}=\varepsilon \dot{x}, \\
\Delta \psi^{(2)}=q \varepsilon \psi^{(1)} .
\end{array}
$$

Moreover, they have the quommutators

$$
\begin{array}{r}
\varepsilon \theta=q^{2} \theta \varepsilon, \\
\theta \psi^{(j)}=q^{j} \psi^{(j)} \theta, \\
\varepsilon \psi^{(j)}=q^{j} \psi^{(j)} \varepsilon .
\end{array}
$$

which let on the same structure as the one present in Section 2. This structure allows us to take the quommutation relation between the two quermionic coordinates, which read

$$
\psi^{(1)} \psi^{(2)}=q^{2} \psi^{(2)} \psi^{(1)} .
$$

Having written down the $q$-superspace transformations and the variations on the $q$-superfield, let us now construct a $q$-covariant derivarive, $D$, that is, a differential operator that obeys

$$
\begin{gathered}
{[D, Q]_{q}=0,} \\
D(\Delta X)=\Delta(D X) .
\end{gathered}
$$


We could try for $D$ the same structure that appears in the $q$-SUSY generator, i.e., to take $D=q^{\alpha} \theta^{2} \partial_{t}+a q^{\beta} \partial_{\theta},(\alpha, \beta=1,2,3 ; a \in C)$. However, it turns out not to be possible to find an operator with this structure and quommuting with $Q$. The only operator that obeys (34) is $Q$ itself, but it obviously does not obey (33).

To construct the coordinates of level 3 permit us to introduce two differential operators, $\partial_{\theta}$ and $\delta_{\theta}$. Using the second one it is possible to show that the operator

$$
D=\theta^{2} \partial_{t}+q \delta_{\theta},
$$

satisfies the conditions (33) and (34).

As in the supersymmetric case, the component fields can be defined by projecting the superfield on different sectors, using the covariant derivatives on $\theta=0$.

$$
\begin{aligned}
\left.X\right|_{\theta=0} & =x \\
\left.D X\right|_{\theta=0} & =\psi^{(2)}, \\
\left.D^{2} X\right|_{\theta=0} & =-\psi^{(1)} .
\end{aligned}
$$

From now on, we will neglect the subscript $\theta=0$.

We also notice some relations between different powers of $D$ and $Q$, that will become useful later

$$
\begin{aligned}
D \cdot \mid & =q^{2} Q \cdot \mid, \\
D^{2} \cdot \mid & =q Q^{2} \cdot \mid, \\
D^{3} \cdot \mid & =-\partial_{t} \cdot \mid .
\end{aligned}
$$

Besides the above-defined bosonic superfield, we can also construct sectors one and two superfields. Their $\theta$ expansion can be taken to be

$$
\Lambda^{(1)}=\lambda^{(1)}+\theta A+q \theta^{2} \lambda^{(2)},
$$

and

$$
\Xi^{(2)}(t)=\xi^{(2)}+q \theta \xi^{(1)}+\theta^{2} F
$$

where the superscripts indicate the sectors to which the fields belongs, and $A$ and $F$ are bosonic fields.

The dimension of the $q$-superfield $\Xi^{(2)}$ is taken to be $\frac{2}{3}$, its bosonic component $F$ being dimensionless and, as we will see later, behaving as an auxiliary field. We cannot, however, take the dimension of the 
$q$-superfield $\Lambda^{(1)}$ to be $\frac{1}{3}$, since this would imply a negative dimension for the component field $\lambda^{(2)}$. Thustake its dimension to be $\frac{4}{3}$. This, however, will produce different equations of motion for its quermionic components, as we will see in the next section.

We assume that the fields $\xi^{(j)}$ have the same behaviour as $\psi^{(j)}$ with respect the quommutations relations with each other, with $\theta$ and with $\varepsilon$.

\section{Examples of Superactions}

In this section, we are going to make a general discussion about simply quadratic actions that are functions of the $q$-superfields introduced in the previous section and give some examples of them.

The action for a generic superfield $\Phi$ must be of the form

$$
S=\int d t d \theta \mathcal{P}\left(\Phi, \dot{\Phi}, D \Phi, D^{2} \Phi\right)
$$

where $\mathcal{P}$ is a polynomial in $\Phi$ and its derivatives. $\mathcal{P}$ must behave like $\theta^{2}$, belonging to the sector two (since $\int d \theta=\partial_{\theta}{ }^{2}$, and $S$ is scalar), and since the measure has mass dimension $\frac{-1}{3}$ and $S$ is dimensionless, its dimension must be $\frac{1}{3}$.

By comparing the expression for the covariant derivative and the $\theta$-integration, we notice the rule

$$
\int d \theta=q^{2} D^{2}
$$

Let us now perform a transformation on the action

$$
\Delta S=\int d t d \theta \Delta \mathcal{P}\left(\Phi, \dot{\Phi}, D \Phi, D^{2} \Phi\right),
$$

since the Jacobian is one, which can be seen by the $t$-independence of the $\theta$ translation. Since $\mathcal{P}$ is a superfield, its variation is of the form of eq.(21). Using this and eq.(41), we arrive at the conclusio

$$
\Delta S=-q \varepsilon \int d t \partial_{t} \mathcal{P}
$$

and the transformations eq.(16) generates symmetries of the action. 
Let us now write an action of the q-superfields $X, \Lambda^{(1)}$ and $\Xi^{(2)}$ defined in Section 3, and compute their equations of motion. We begin with the bosonic superfield $X$. Its quadratic action is

$$
S_{X}=-\frac{m}{2} \int d t d \theta q^{2}\left(D^{2} X\right)\left(D^{2} X\right),
$$

where $m$ is a commuting mass parameter. By explicit computation of its $\theta$ integral, or by use of eq.(41), this action can be writen down in in components as

$$
S_{X}=m \int d t\left(\frac{1}{2} \dot{x}^{2}-2 q \dot{\psi}^{(2)} \psi^{(1)}\right),
$$

where the difference with the Section 1 action is due to the different initial superactions in these cases.

Although the variational calculus of the quermionic coordinates presents several difficulties to overcome (for instance, how to do the variation with respect to a quermion), it is clear that the equation of motion arising from the above Lagrangian is, up to multiplicative factors $D \dot{X}=0$, giving in components $\ddot{x}=\dot{\psi}^{(j)}=0(j=1,2)$. Computing its $q$-supersymmetric variation, we obtain

$$
\Delta S_{X}=q \epsilon \int d t \frac{\partial \psi^{(1)^{2}}}{\partial t}
$$

We notice that the action given by eq.(44), its variation eq.(46) and the variation of the component fields eq.(30) are, up to factors, equal to eqs.(12), (15) and (14) respectively, recalling that the presence of such cocicle-type factors was because the quommutation homogeneity assumption had been used. Thus we see that the $q$-superfield $X$ describes the dynamics of a free particle partners.

The quadratic action for the $q$-superfield $\Lambda^{(1)}$ is

$$
S_{\Lambda}=-\frac{m}{2} \int d t d \theta\left(\dot{\Lambda}^{(1)}\right)^{2} .
$$

By convenience the mass parameter was taken to be the same as in the $X$ action. In components, the action turns out to be

$$
S_{\Lambda}=\frac{m}{2} \int d t\left(\dot{A}^{2}+2 q \dot{\lambda}^{(2)} \dot{\lambda}^{(1)}\right) .
$$

It is interesting to notice that the equation of motion for $\Lambda^{(1)}$, obtained from its action, $\ddot{\Lambda}^{(1)}=0$, gives in component $\ddot{A}=\ddot{\lambda}^{(i)}=0$. Thus this 
$q$-superfield also represents a free particle, but its quermionic partners obey an equation of motion that is of second order in the time derivative, whereas in the case of $q$-superfield $X$ it is of first order. The $q$-supersymmetric variation of the $S_{\Lambda}$ is

$$
\Delta S_{\Lambda}=\varepsilon \int d t \frac{\partial\left(\dot{\Lambda}^{(1)}\right)^{2}}{\partial t}
$$

We now consider the quadratic action for the q-superfield $\Xi^{(2)}$. It is

$$
S_{\Xi}=m \int d t d \theta\left(D \Xi^{(2)}\right)^{2} .
$$

In component fields, the action reads

$$
S_{\Xi}=m \int d t\left[2 q \dot{\xi^{(2)}} \xi^{(1)}+F^{2}\right] .
$$

The equation of motion for $\Xi^{(2)}$ is $D^{2} \Xi^{(2)}=0$, giving $F=\dot{\xi}^{(j)}=0$, meaning, as it was anticipated, that the bosonic coordinate $F$ is an auxiliary one. The variation of $S_{\Xi}$ is

$$
\Delta S_{\Xi}=-\epsilon \int d t \frac{\partial \xi^{(1)^{2}}}{\partial t} .
$$

The superfields $X$ and $\Xi^{(2)}$ can have a quadratic action with a mixed term

$$
S_{X \Xi}=m \omega \int d t d \theta q^{2} X \Xi^{(2)}, l
$$

where $\omega$ has a mass ${ }^{-1}$ dimension. In components we write this action as

$$
S_{X \Xi}=m \omega \int d t\left(F x+q^{2} \psi^{(1)} \xi^{(2)}+q \psi^{(2)} \xi^{(1)}\right) .
$$

Summing up the actions (44), (50) and (54) $S_{H O}=S_{X}+S_{\Xi}+S_{X \Xi}$, and its bosonic part is

$$
S_{H O}=\int d t m\left(\frac{1}{2} \dot{x}^{2}+\frac{1}{2} F^{2}+\omega F x\right) .
$$

Computing the equation of motion of the auxiliary field $F$ and reintroducing it in the action, it becomes

$$
S_{x}=\int d t\left[\frac{1}{2} m \dot{x}^{2}-\frac{1}{2} m \omega x\right],
$$

which is the action for the harmonic oscillator. 


\section{Conclusions}

In this letter, we presented a generalization of some supersymmetric classical mechanical models where the superspace has a non-commuting coordinate nilpotent of order 3 , and the commutation relations among the several objects of the model are deformed by powers of a parameter $q$. Translations on the $q$-superspace induce transformations on the fields, and the operatorial realization of the supersymmetric generator is obtained by a suitable Taylor expansion. The covariant derivative was also introduced, in which we used a second kind of partial paragrassmannian derivative. Spite the supersymmetric structure similarity, we are facing a slightly different situation. In fact because of the presence of two derivatives, like the forward and the backward one, it resembles a lattice approach. In a very recent and nice work [13], the authors showed the rôles playing by the covariant derivative $D$ and the symmetry generator $Q$, present in this work, are the left and right action of $G_{3}$ group.

After introducing superfields belonging to different sectors, we were able to construct quadratic actions for each one. These actions are, up to total derivatives, invariant under the $q$-supersymmetric transformations. Using a naïve approach, it is possible to extract from these actions the equations of motion since there is no, up to now, a well-defined differential calculus on these quermionic coordinates. We intend to discuss this subject in a forthcoming publication. We also showed that imposing the "on-shell" constraint to the auxiliary fields, it is possible to get the harmonic oscillator as a bosonic sector of a simple suitable linear combination of the actions.

It should also be interesting to study this formulation from a field theoretical point of view, in particular in the $(2+1)$-dimensional case. We might also try to understand if such fields are representations of some $q$-deformed algebra, either a $q$-Poincaré or a $q$-Clifford one.

Acknowledgements: The authors would like to thank J.A. Helayël-

Neto and Marco A. R. Monteiro for discussions. The authors also thanks to CNPq and FAPERJ for financial support and for the DCP/CBPF hospitality. 


\section{References}

[1] E.K. Sklyanin and L.D. Fadeev, Sov. Phys. Dokl. 23,(1978) 902,

[2] V.G. Drinfeld, Sov. Math. Dokl. 27 (1983) 68,

[3] S.L. Woronowicz, Comm. Math. Phys. 111 (1987) 613,

[4] J.Wess and B. Zumino, Covariant differential calculus on the quantum hyperplane, Preprint CERN-TH-5697/90, LAPP-TH$284 / 90$,

[5] A.J. Macfarlane, J. Phys. A Math. Gen. 22 (1989) 4581, L.C. Biedernharn, J. Phys. A22 (1989) L873,

[6] L. Baulieu and E. Floratos, Phys. Lett. B258 (1991) 271,

[7] J.L. Matheus-Valle and Marco A.R. Monteiro, Mod. Phys. Lett. A7 (1992) 3023,

[8] J.L. Matheus-Valle and Marco A.R. Monteiro, Phys. Lett. B300 (1993) 66,

[9] A.T. Filipov, A.P. Isaev and A.B. Kurdikov, Mod. Phys. Lett. 7, (1992) 2129,

[10] C. Ahn, D. Bernard and A. LeClair, Nucl. Phys. B346 (1990) 409 ,

[11] S. Durand, Mod. Phys. Lett. A8 (1993) 2323,

[12] N. Debergh, J. Phys. A26 (1993) 7219,

N. Mohammedi, Fractional Supersymmetry, hep-th/9412133,

[13] J.A. de Azcárraga and A.J. Macfarlane, Group Theoretica Foundations of Fractional Supersymmetry, preprint FTUV/95-23, hep-th 9506185. 